\title{
Choice of Sampling Interval and Extent for Finite-Energy Fields
}

\author{
Talha Cihad Gulcu, Student Member, IEEE, and Haldun M. Ozaktas, Fellow, IEEE
}

\begin{abstract}
We focus on the problem of representing a nonstationary finite-energy random field, with finitely many samples. We do not require the field to be of finite extent or to be bandlimited. We propose an optimizable procedure for obtaining a finite-sample representation of the given field. We estimate the reconstruction error of the procedure, showing that it is the sum of the truncation errors in the space and frequency domains. We also optimize the truncation parameters analytically and present the resultant Pareto-optimal tradeoff curves involving the error in reconstruction and the sample count, for several examples. These tradeoff curves can be used to determine the optimal sampling strategy in a practical situation based on the relative importance of error and sample count for that application.
\end{abstract}

Index Terms-Random field estimation, finite-energy signals, non-stationary signals, uniform sampling, reconstruction error.

\section{INTRODUCTION}

I $\mathrm{N}$ THIS work, we present a method to represent any nonstationary finite-energy field by using finitely many samples. This problem is not only practical, but also has theoretical and conceptual significance. We analytically characterize the source of the inevitable approximation and error involved, how this error is related to our choice of the fundamental parameters, and the tradeoff between representational accuracy and efficiency. More specifically, we analyze the reconstruction error, and see that it is approximately equal to the energy of the truncated parts of the signal lying outside the chosen space and frequency extents, and that it can be made as small as we wish by choosing these extents large enough. By optimizing the space and frequency extents, we obtain the best possible (Pareto-optimal) tradeoff curves involving the reconstruction error and the sample count. This gives us a very satisfying picture of what it means to represent a function using a finite number of samples.

We can only give a very brief survey of works on sampling of a field. Review articles on sampling include [1]-[4]. Some extensions of the Shannon-Nyquist theorem are presented

Manuscript received May 27, 2016; revised October 28, 2016 and December 8, 2016; accepted December 8, 2016. Date of publication December 19, 2016; date of current version January 27, 2017. The associate editor coordinating the review of this manuscript and approving it for publication was Prof. Masahiro Yukawa. The work of H. M. Ozaktas was supported in part by the Turkish Academy of Sciences.

T. C. Gulcu was with the Department of Electrical \& Computer Engineering, University of Maryland, College Park, MD 20742 USA. He is now with TUBITAK Advanced Technologies Research Institute, 06800 Ankara, Turkey (e-mail: gulcu@umd.edu).

H. M. Ozaktas is with the Department of Electrical Engineering, Bilkent University, Ankara TR-06800, Turkey (e-mail: haldun@ee.bilkent.edu.tr).

Color versions of one or more of the figures in this paper are available online at http://ieeexplore.ieee.org.

Digital Object Identifier 10.1109/TSP.2016.2641391 in [1], including sampling of functions of more than one variable, random processes, non-bandlimited functions, generalized functions; as well as nonuniform sampling, implicit sampling, sampling with derivatives, and sampling for general integral transforms. A review of sampling theory for non-bandlimited signals is given in [2], covering sup-norm error for bandlimiting operations, and sampling approximation of derivatives. The main focus of [3] is uniform sampling with more general classes of interpolating functions and wider classes of anti-aliasing prefilters. Non-bandlimited signal recovery from nonuniform samples and pointwise reconstruction stability are reviewed in [4]. Sampling theorem for bandlimited random processes was considered in [5]. Reference [6] provides an error analysis for nonuniform sampling of non-bandlimited signals that are timelimited. A different error analysis addressing the aliasing effect for uniform sampling of non-bandlimited signals appears in [7], providing a counterexample on how much the 2-norm of the aliasing error can be. While [7] provides an analysis of 2-norm truncation error, there are other works, all dealing with bandlimited signals [8]-[10] focusing on the sup-norm of the error term. A recent paper deals with variable-density sampling strategies for compressive imaging [11]. References [12]-[15] focus on the subject of reconstruction from irregular samples. Reference [12] covers the approximation of some convolution operators and the representation of signals in a wide class of function spaces and in general locally compact abelian groups by irregular samples. Reconstruction of a bandlimited function from any random sampling set is considered and the rate of convergence for the proposed iteration method is estimated in [13], while exact reconstruction of spline-like functions from irregular samples using an iterative fast algorithm is dealt with in [14]. Analysis of the maximum deviation from regularity that can be tolerated to ensure exact recovery for bandlimited functions is the subject of [15]. Tradeoffs related to the accuracy of the sample measurements are considered in [16]-[18]. A brute-force method for optimal reconstruction from uniform samples is investigated in [19]. Wavelets and splines are considered in numerous works [20]-[22] as the interpolating function. In [20], perfect reconstruction filter banks are reviewed. For a low-pass filter, the necessary and sufficient conditions for the existence of a complementary high-pass filter that will permit perfect reconstruction are derived. A family of nonorthogonal polynomial spline wavelet transform is introduced and analyzed in [21] while [22] presents the derivation of filtering algorithms for polynomial spline interpolation and the construction of an extended family of polynomial spline wavelet transforms.

As for the study of non-stationary signals, we can only mention a small sample of recent papers [23]-[27]. Reference [28] is a special issue dedicated to the subject. Several works have dealt with sampling of non-stationary signals [29]-[31]. In particular, 
[29] shows that mean-square error goes to zero for all time instants when the non-stationary process of interest is bandlimited.

Another concept highly relevant to the present work is the number of degrees of freedom (DOF) of a field. This is a fundamental concept appearing in many disciplines, especially those dealing with propagating wave signals, such as optics, electromagnetics, and wireless communications [32]-[41]. The DOF is sometimes, but not always, the same as the space-bandwidth or time-bandwidth product [42], [43].

We emphasize that in our framework, both the energy of the field and the total number of samples used is finite, as opposed to the common case of considering the number of samples per unit interval to be finite for infinite-energy processes or fields [44], [45]. We aim to develop a thorough understanding of the nature and tradeoffs involved in the finite sampling and sinc interpolation problem in its most basic form. To this end, we strive to not deviate from an analytical development, and succeed in solving the optimal sampling problem analytically. Our approach is sufficient to address important basic problems such as determining the minimum number of samples required to achieve a specified error tolerance, finding the optimal sampling frequency and the optimal sampling extent, and determining the minimum representation error for a given number of samples.

The minimum number of samples required to represent and recover a random field may be interpreted as the number of degrees of freedom of the field. So the method of our paper allows us to find the number of degrees of freedom of a field from its autocorrelation function. It is important to underline that this also depends on the specified error tolerance in a fundamental way. This is most obviously seen if we consider how the space-bandwidth (or time-bandwidth) product of a signal is roughly estimated by simply multiplying the extents of the signal in the space (or time) and frequency domains. Since it is a fundamental fact that both extents cannot be finite, determination of these finite extents involves a decision regarding how much of the tails of the functions can be neglected. This decision is sometimes explicit but often implicit. Thus the number of degrees of freedom of a signal is not an absolute concept but is conditioned on the error tolerance. These considerations and relationships are put on solid ground in this paper.

The rest of the paper is organized as follows. In Sections II and III, we give the problem formulation and the reconstruction error analysis, respectively. Reconstruction without prefiltering is discussed in Section IV. Optimization of the sampling variables is explained in Section V. The numerical results are provided in Section VI. We conclude the paper in Section VII.

\section{Finite-SAMPle RePRESENTATION OF FINITE-ENERGY FIELDS}

In this section, we explain how we represent a given finiteenergy field with finitely many samples. Our representation has two parameters: Spatial extent $\Delta u$ and spectral extent $\Delta \mu$. We will show that the error in such a representation is approximately equal to the energy of the signal falling outside of these two extents (Theorem 2), and that it can be made as small as we wish by choosing the extents sufficiently large (Theorem 1).

Let $f: \mathbb{R} \rightarrow \mathbb{C}$ be a finite-energy field having continuous realizations with the covariance function $K_{f}\left(u_{1}, u_{2}\right)=$
$E\left[f\left(u_{1}\right) f^{*}\left(u_{2}\right)\right]$. The finite-energy assumption means that

$$
\begin{aligned}
E\left[\int_{-\infty}^{\infty}|f(u)|^{2} d u\right] & =\int_{-\infty}^{\infty} E\left[|f(u)|^{2}\right] d u \\
& =\int_{-\infty}^{\infty} K_{f}(u, u) d u<\infty
\end{aligned}
$$

We are able to change the order of integration and expectation in (1) using Fubini-Tonelli's theorem and the fact that $f$ has to be product measurable [46], [47].

In addition to the conditions we have stated, we will also assume that the Fourier transform exists for almost all realizations of $f$. Let $F: \mathbb{R} \rightarrow \mathbb{C}$ denote the Fourier transform of $f$. We wish to sample the bandlimited version of $f$ having the Fourier transform

$$
F_{\Delta \mu}(\mu)=\left\{\begin{aligned}
F(\mu), & \text { if }|\mu| \leq \Delta \mu / 2 \\
0, & \text { else }
\end{aligned}\right.
$$

to avoid aliasing. Let

$$
f_{\Delta \mu}(u)=\int_{-\Delta \mu / 2}^{\Delta \mu / 2} F(\mu) e^{j 2 \pi u \mu} d \mu
$$

denote the resultant signal once the spectral content of $f$ outside of the interval $[-\Delta \mu / 2, \Delta \mu / 2]$ has been discarded. From the sampling theorem, we have

$$
f_{\Delta \mu}(u)=\sum_{n=-\infty}^{\infty} f_{\Delta \mu}\left(\frac{n}{\Delta \mu}\right) \operatorname{sinc}(\Delta \mu u-n)
$$

In order to represent the initial signal $f$ with finitely many samples, we discard the samples $f_{\Delta \mu}\left(\frac{n}{\Delta \mu}\right)$ lying outside $[-\Delta u / 2, \Delta u / 2]$. The new signal we obtain is denoted by $f_{\Delta \mu, \Delta u}(u)$ and is given by

$$
f_{\sim \mu, \Delta u}(u)=\sum_{n=-\lfloor\Delta u \Delta \mu / 2\rfloor}^{\lfloor\Delta u \Delta \mu / 2\rfloor} f_{\sim \mu}\left(\frac{n}{\Delta \mu}\right) \operatorname{sinc}(\Delta \mu u-n)
$$

which can be characterized completely by the following number of samples:

$$
2\left\lfloor\frac{\Delta u \Delta \mu}{2}\right\rfloor+1 \approx \Delta u \Delta \mu
$$

which is commonly known as the space-bandwidth or timebandwidth product [42], [43]. The samples defining $f_{\Delta \mu, \Delta u}$ as in (2) constitute the vector

$$
\mathbf{f}=\left(f_{\Delta \mu}\left(\frac{n}{\Delta \mu}\right) \mid-\left\lfloor\frac{\Delta u \Delta \mu}{2}\right\rfloor \leq n \leq\left\lfloor\frac{\Delta u \Delta \mu}{2}\right\rfloor\right)
$$

which is the finite-sample representation of $f$.

Before continuing we note that the subscripts $\Delta u$ and $\Delta \mu$ denote the extents of truncation in the space and frequency domains. The under tilde indicates that the truncation has been done in the domain opposite to which the signal is being represented in. For example, the Fourier transform of $f_{\Delta \mu, \Delta u}(u)$ is denoted as $F_{\Delta \mu, \Delta u}(\mu)$.

The dual of the representation described above can be obtained by truncating $f$ in the spatial domain, and sampling its 
Fourier transform. More precisely, we define the signal

$$
f_{\Delta u}(u)=\left\{\begin{aligned}
f(u), & \text { if }|u| \leq \Delta u / 2 \\
0, & \text { else }
\end{aligned}\right.
$$

whose Fourier transform can be expressed as

$$
F_{\sim}(\mu)=\sum_{n=-\infty}^{\infty} F_{\Delta u}\left(\frac{n}{\Delta u}\right) \operatorname{sinc}(\Delta u \mu-n)
$$

using the sampling theorem. Keeping the samples $F_{\Delta u}\left(\frac{n}{\Delta u}\right)$
which belong to the frequency band $[-\Delta \mu / 2, \Delta \mu / 2]$ only, we obtain the signal

$$
F_{\Delta u, \Delta \mu}(\mu)=\sum_{n=-\lfloor\Delta u \Delta \mu / 2\rfloor}^{\lfloor\Delta u \Delta \mu / 2\rfloor} F_{\Delta u}\left(\frac{n}{\Delta u}\right) \operatorname{sinc}(\Delta u \mu-n) .
$$

$F_{\Delta u, \Delta \mu}(\mu)$ is uniquely defined by $2\left\lfloor\frac{\Delta u \Delta \mu}{2}\right\rfloor+1 \approx \Delta u \Delta \mu$ samples, and its inverse Fourier transform $f_{\Delta u, \Delta \mu}$ can be used to recover $f$ as an alternative to $f_{\Delta \mu, \Delta u}$ defined by (2).

We now analyze the error made by reconstructing $f(u)$ as $f_{\Delta \mu, \Delta u}(u)$. For that purpose, we write

$$
\begin{aligned}
& \left|f(u)-f_{\Delta \mu, \Delta u}(u)\right|^{2} \\
& =\left|\left(f(u)-f_{\sim_{\mu}}(u)\right)+\left(f_{\underset{\sim}{\sim}}(u)-f_{\underset{\sim}{\Delta}, \Delta u}(u)\right)\right|^{2} \\
& =\left|f(u)-f_{\Delta \mu}(u)\right|^{2} \\
& +2 \operatorname{Re}\left\{\left(f(u)-f_{\Delta \mu}(u)\right)\left(f_{\Delta \mu}(u)-f_{\sim}{ }_{\sim}, \Delta u(u)\right)^{*}\right\} \\
& +\left|f_{\Delta \mu}(u)-f_{\underset{\sim}{\Delta}, \Delta u}(u)\right|^{2}
\end{aligned}
$$

which implies

$$
\begin{aligned}
& \int_{-\infty}^{\infty}\left|f(u)-f_{\Delta \mu, \Delta u}(u)\right|^{2} d u=\int_{-\infty}^{\infty}\left|f(u)-f_{\Delta \mu}(u)\right|^{2} d u \\
& +2 \operatorname{Re}\left\{\left\langle f(u)-f_{\Delta \mu}(u), f_{\Delta \mu}(u)-f_{\Delta \mu, \Delta u}(u)\right\rangle\right\} \\
& \quad+\int_{-\infty}^{\infty}\left|f_{\Delta \mu}(u)-f_{\Delta \mu}, \underset{\sim}{\sim}(u)\right|^{2} d u
\end{aligned}
$$

where $\langle.,$.$\rangle denotes the inner product, defined by$

$$
\langle f(u), g(u)\rangle=\int_{-\infty}^{\infty} f(u) g^{*}(u) d u
$$

Since Fourier transform preserves inner products, we have

$$
\begin{aligned}
& \left\langle f(u)-f_{\sim \mu}(u), f_{\sim_{\mu}}(u)-f_{\sim_{\mu}, \Delta u}(u)\right\rangle \\
& \quad=\left\langle F(\mu)-F_{\Delta \mu}(\mu), F_{\Delta \mu}(\mu)-F_{\Delta \mu, \Delta u}(\mu)\right\rangle
\end{aligned}
$$

from which

$$
\begin{aligned}
& \left\langle f(u)-f_{\sim_{\mu}}(u), f_{\sim_{\mu}}(u)-f_{\Delta \mu, \Delta u}(u)\right\rangle \\
& =\int_{-\Delta \mu / 2}^{\Delta \mu / 2}\left(F(\mu)-F_{\Delta \mu}(\mu)\right)\left(F_{\Delta \mu}(\mu)-F_{\Delta \mu, \Delta u}(\mu)\right)^{*} d \mu \\
& \quad+\int_{|\mu|>\Delta \mu / 2}\left(F(\mu)-F_{\Delta \mu}(\mu)\right)\left(F_{\Delta \mu}(\mu)-F_{\Delta \mu, \Delta u}(\mu)\right)^{*} d \mu
\end{aligned}
$$$$
=\int_{-\Delta \mu / 2}^{\Delta \mu / 2} 0\left(F_{\Delta \mu}(\mu)-F_{\Delta \mu, \Delta u}(\mu)\right)^{*} d \mu
$$$$
+\int_{|\mu|>\Delta \mu / 2}\left(F(\mu)-F_{\Delta \mu}(\mu)\right) 0 d \mu=0+0=0
$$

follows. Thus, (6) takes the form

$$
\begin{aligned}
& \int_{-\infty}^{\infty}\left|f(u)-f_{\sim} \underset{\sim}{\mu, \Delta u}(u)\right|^{2} d u=\int_{-\infty}^{\infty}\left|f(u)-f_{\sim_{\mu}}(u)\right|^{2} d u \\
& +\int_{-\infty}^{\infty}\left|f_{\Delta \mu}(u)-f_{\sim_{\mu}, \Delta u}(u)\right|^{2} d u \\
& =\int_{|\mu|>\Delta \mu / 2}|F(\mu)|^{2} d \mu+\int_{-\infty}^{\infty}\left|f_{\Delta \mu}(u)-f_{\Delta \mu, \Delta u}(u)\right|^{2} d u .
\end{aligned}
$$

To simplify $\int_{-\infty}^{\infty}\left|f_{\Delta \mu}(u)-f_{\Delta \mu}, \Delta u(u)\right|^{2} d u$, we first write

$$
\begin{aligned}
& \int_{-\infty}^{\infty} \mid f_{\Delta \mu}(u)-f_{\sim} \underset{\sim}{\sim}, \Delta u \\
& =\int_{-\infty}^{\infty}\left|\sum_{|n|>\lfloor\Delta u \Delta \mu / 2\rfloor} f_{\Delta \mu}\left(\frac{n}{\Delta \mu}\right) \operatorname{sinc}(\Delta \mu u-n)\right|^{2} d u
\end{aligned}
$$

which can be expressed as

$$
\int_{-\infty}^{\infty}\left|\lim _{k \rightarrow \infty} \sum_{\lfloor\Delta u \Delta \mu / 2\rfloor<|n| \leq k} f_{\Delta \mu}\left(\frac{n}{\Delta \mu}\right) \operatorname{sinc}(\Delta \mu u-n)\right|^{2} d u
$$

We now take the limit outside the integral (this will be justified further below):

$$
\begin{aligned}
\lim _{k \rightarrow \infty} & {\left[\int_{-\infty}^{\infty}\left|\sum_{\lfloor\Delta u \Delta \mu / 2\rfloor<|n| \leq k} \underset{\sim}{f_{\Delta \mu}}\left(\frac{n}{\Delta \mu}\right) \operatorname{sinc}(\Delta \mu u-n)\right|^{2} d u\right] } \\
= & \lim _{k \rightarrow \infty} \sum_{\lfloor\Delta u \Delta \mu / 2\rfloor<\left|k_{1}\right| \leq k} \sum_{\lfloor\Delta u \Delta \mu / 2\rfloor<\left|k_{2}\right| \leq k} f_{\Delta \mu}\left(\frac{k_{1}}{\Delta \mu}\right) \\
& \cdot f_{\Delta \mu}^{*}\left(\frac{k_{2}}{\Delta \mu}\right)\left\langle\operatorname{sinc}\left(\Delta \mu u-k_{1}\right), \operatorname{sinc}\left(\Delta \mu u-k_{2}\right)\right\rangle .
\end{aligned}
$$


Noting that the inner product term $\left\langle\operatorname{sinc}\left(\Delta \mu u-k_{1}\right)\right.$, $\left.\operatorname{sinc}\left(\Delta \mu u-k_{2}\right)\right\rangle \operatorname{simplifies~to~}$

$$
\begin{aligned}
& \left\langle\operatorname{sinc}\left(\Delta \mu u-k_{1}\right), \operatorname{sinc}\left(\Delta \mu u-k_{2}\right)\right\rangle \\
& =\left\langle\frac{1}{\Delta \mu} \operatorname{rect}\left(\frac{\mu}{\Delta \mu}\right) e^{-j 2 \pi \frac{k_{1}}{\Delta \mu} \mu}, \frac{1}{\Delta \mu} \operatorname{rect}\left(\frac{\mu}{\Delta \mu}\right) e^{-j 2 \pi \frac{k_{2}}{\Delta \mu} \mu}\right\rangle \\
& =\frac{1}{\Delta \mu} \delta_{k_{1} k_{2}},
\end{aligned}
$$

where $\delta_{k_{1} k_{2}}$ is the Kronecker delta ( $=1$ when $k_{1}=k_{2}$ and $=0$ otherwise), we get

$$
\begin{aligned}
& \lim _{k \rightarrow \infty} \int_{-\infty}^{\infty}\left|\sum_{\lfloor\Delta u \Delta \mu / 2\rfloor<|n| \leq k} f_{\Delta \mu}\left(\frac{n}{\Delta \mu}\right) \operatorname{sinc}(\Delta \mu u-n)\right|^{2} d u \\
& =\lim _{k \rightarrow \infty} \sum_{\lfloor\Delta u \Delta \mu / 2\rfloor<|n| \leq k} \frac{1}{\Delta \mu}\left|f_{\Delta \mu}\left(\frac{n}{\Delta \mu}\right)\right|^{2}
\end{aligned}
$$

The expression (12) has to be finite with probability 1 since

$$
\begin{aligned}
\sum_{\mid \Delta u \Delta \mu / 2\rfloor<|n| \leq k} \frac{1}{\Delta \mu}\left|f_{\Delta \mu}\left(\frac{n}{\Delta \mu}\right)\right|^{2} & \leq \int_{-\infty}^{\infty}\left|f_{\Delta \mu}(u)\right|^{2} d u \\
& \leq \int_{-\infty}^{\infty}|f(u)|^{2} d u
\end{aligned}
$$

holds true for all $k \in \mathbb{N}$. (Note that the first inequality in (13) follows from the equation

$$
\begin{array}{r}
\left\langle f_{\sim \mu}(u)-\sum_{\lfloor\Delta u \Delta \mu / 2\rfloor<|n| \leq k} f_{\Delta \mu}\left(\frac{n}{\Delta \mu}\right) \operatorname{sinc}(\Delta \mu u-n),\right. \\
\left.\sum_{\lfloor\Delta u \Delta \mu / 2\rfloor<|n| \leq k} f_{\Delta \mu}\left(\frac{n}{\Delta \mu}\right) \operatorname{sinc}(\Delta \mu u-n)\right\rangle=0,
\end{array}
$$

which is a consequence of (11).)

Knowing that the limit expression (12) and hence (10) is finite allows us to invoke the dominated convergence theorem [46], which justifies taking the limit outside the integral following (10). Consequently, (10) simplifies to

$$
\begin{aligned}
& \int_{-\infty}^{\infty}\left|f_{\Delta \mu}(u)-f_{\Delta \mu, \Delta u}(u)\right|^{2} d u \\
& =\sum_{\lfloor\Delta u \Delta \mu / 2\rfloor<|n|} \frac{1}{\Delta \mu}\left|f_{\Delta \mu}\left(\frac{n}{\Delta \mu}\right)\right|^{2} .
\end{aligned}
$$

Inserting (14) in (9), we obtain

$$
\begin{aligned}
& \int_{-\infty}^{\infty}\left|f(u)-f_{\Delta \mu, \Delta u}(u)\right|^{2} d u \\
& =\int_{|\mu|>\Delta \mu / 2}|F(\mu)|^{2} d \mu+\sum_{\lfloor\Delta u \Delta \mu / 2\rfloor<|n|} \frac{1}{\Delta \mu}\left|f_{\Delta \mu}\left(\frac{n}{\Delta \mu}\right)\right|^{2}
\end{aligned}
$$

The dual expression

$$
\begin{aligned}
& \int_{-\infty}^{\infty}\left|f(u)-f_{\Delta u, \Delta \mu}(u)\right|^{2} d u \\
& =\int_{|u|>\Delta u / 2}|f(u)|^{2} d u+\sum_{\lfloor\Delta u \Delta \mu / 2\rfloor<|n|} \frac{1}{\Delta u}\left|F_{\Delta u}\left(\frac{n}{\Delta u}\right)\right|^{2}
\end{aligned}
$$

follows similarly. Taking the expectation of both sides in (15), and defining the spectral covariance as $S_{f}\left(\mu_{1}, \mu_{2}\right)=E\left[F\left(\mu_{1}\right) F^{*}\left(\mu_{2}\right)\right]$ we get

$$
\begin{aligned}
& E \int_{-\infty}^{\infty}\left|f(u)-f_{\sim \mu, \Delta u}(u)\right|^{2} d u \\
& =E \int_{|\mu|>\Delta \mu / 2}|F(\mu)|^{2} d \mu+E \sum_{\lfloor\Delta u \Delta \mu / 2\rfloor<|n|} \frac{1}{\Delta \mu}\left|f_{\Delta \mu}\left(\frac{n}{\Delta \mu}\right)\right|^{2} \\
& =\int_{|\mu|>\Delta \mu / 2} S_{f}(\mu, \mu) d \mu+E \sum_{\lfloor\Delta u \Delta \mu / 2\rfloor<|n|} \frac{1}{\Delta \mu}\left|f_{\Delta \mu}\left(\frac{n}{\Delta \mu}\right)\right|^{2}
\end{aligned}
$$

Equation (1) implies $\lim _{\Delta \mu \rightarrow \infty} \int_{|\mu|>\Delta \mu / 2} S_{f}(\mu, \mu) d \mu=0$, and inequality (13) implies

$$
\begin{aligned}
\lim _{\Delta u \rightarrow \infty} E\left[\sum_{\lfloor\Delta u \Delta \mu / 2\rfloor<|n|} \frac{1}{\Delta \mu}\left|f_{\Delta \mu}\left(\frac{n}{\Delta \mu}\right)\right|^{2}\right] \\
=\lim _{\Delta u \rightarrow \infty} \sum_{\lfloor\Delta u \Delta \mu / 2\rfloor<|n|} \frac{1}{\Delta \mu} E\left|f_{\Delta \mu}\left(\frac{n}{\Delta \mu}\right)\right|^{2}=0 .
\end{aligned}
$$

Combining these two facts with (17), we see that the average reconstruction error $E \int_{-\infty}^{\infty}\left|f(u)-f_{\Delta \mu, \Delta u}(u)\right|^{2} d u$ can be made arbitrarily small by choosing the spatial extent $\Delta u$ and the spectral extent $\Delta \mu$ sufficiently large. Proceeding in a similar way, from (16) we obtain

$$
\begin{aligned}
& E \int_{-\infty}^{\infty}\left|f(u)-f_{\Delta u, \Delta \mu}(u)\right|^{2} d u \\
& =\int_{|u|>\Delta u / 2} K_{f}(u, u) d u+E \sum_{\lfloor\Delta u \Delta \mu / 2\rfloor<|n|} \frac{1}{\Delta u}\left|F_{\Delta u}\left(\frac{n}{\Delta u}\right)\right|^{2}
\end{aligned}
$$

which implies the average error $E \int_{-\infty}^{\infty}\left|f(u)-f_{\Delta u, \Delta \mu}(u)\right|^{2} d u$ goes to zero as $\Delta u$ and $\Delta \mu$ tends to infinity. These results are summarized in the following theorem.

Theorem 1: Let $f: \mathbb{R} \rightarrow \mathbb{C}$ be a random field having covariance function $K_{f}\left(u_{1}, u_{2}\right)=E\left[f\left(u_{1}\right) f^{*}\left(u_{2}\right)\right]$ with continuous realizations, and let $f$ be a finite energy field in the sense that $\int_{-\infty}^{\infty} E|f(u)|^{2} d u=\int_{-\infty}^{\infty} K_{f}(u, u) d u<\infty$.

Then, the reconstruction $f_{\Delta \mu, \Delta u}(u)$ given in (2) can be obtained from $\Delta u \Delta \mu \pm 1$ (see (3)) samples, such that the average reconstruction error $E \int_{-\infty}^{\infty}\left|f(u)-f_{\Delta \mu, \Delta u}(u)\right|^{2} d u$ goes to zero as $\Delta u, \Delta \mu \rightarrow \infty$. A similar result is valid for the reconstruction $f_{\Delta u, \Delta \mu}(u)$.

This is an important observation regarding the representation of finite-energy signals with finitely many samples. It is well known that a function and its Fourier transform cannot both be 
confined within a finite extent, so that for given finite $\Delta u$ and $\Delta \mu$, there will always be some representation and reconstruction error. However, this error can be made arbitrarily small by increasing $\Delta u$ and $\Delta \mu$. We now proceed to show that this error can be characterized in a very simple and intuitive manner.

\section{ERROR ANALYSIS OF FINITE-SAMPLE REPRESENTATION}

To analyze the reconstruction error $\int_{-\infty}^{\infty} \mid f(u)-$ $\left.f_{\Delta \mu, \Delta u}(u)\right|^{2} d u$, we focus on the summation term appearing in $\sim(15)$. Since $f_{\Delta \mu}(u)$ is bandlimited to $[-\Delta \mu / 2, \Delta \mu / 2]$, the value it takes doẽs not fluctuate significantly over a length of $1 / \Delta \mu$. Therefore, we may assume that the approximation

$$
\begin{aligned}
& \sum_{\lfloor\Delta u \Delta \mu / 2\rfloor<|n|} \frac{1}{\Delta \mu}\left|f_{\Delta \mu}\left(\frac{n}{\Delta \mu}\right)\right|^{2} \\
& \approx \int_{|u|>\frac{\lfloor\Delta u \Delta \mu / 2\rfloor}{\Delta \mu}}\left|f_{\Delta \mu}(u)\right|^{2} d u \approx \int_{|u|>\Delta u / 2}\left|f_{\Delta \mu}(u)\right|^{2} d u
\end{aligned}
$$

is valid for most of the realizations (with a high probability). Alternatively, one can argue that

$$
\begin{gathered}
\int_{|u|>\frac{\lfloor\Delta u \Delta \mu / 2\rfloor+1}{\Delta \mu}}\left|f_{\Delta \mu}(u)\right|^{2} d u \\
\leq \sum_{\lfloor\Delta u \Delta \mu / 2\rfloor<|n|} \frac{1}{\Delta \mu}\left|f_{\Delta \mu}\left(\frac{n}{\Delta \mu}\right)\right|^{2} \\
\quad \leq \int_{|u|>\frac{\lfloor\Delta u \Delta \mu / 2\rfloor}{\Delta \mu}}\left|f_{\Delta \mu}(u)\right|^{2} d u
\end{gathered}
$$

provided $\left|f_{\Delta \mu}(u)\right|^{2}$ is monotone decreasing for $u>\Delta u / 2$ and

increasing for $u<-\Delta u / 2$, which is expected to be the case with a high probability if $\Delta u$ is large enough. Combining the observations given by (19) and (20) with (15), we get

$$
\begin{aligned}
& \int_{-\infty}^{\infty}\left|f(u)-f_{\Delta \mu, \Delta u}(u)\right|^{2} d u \\
& \quad \approx \int_{|\mu|>\Delta \mu / 2}|F(\mu)|^{2}+\int_{|u|>\Delta u / 2}\left|f_{\Delta \mu}(u)\right|^{2} d u \\
& \quad \approx \int_{|\mu|>\Delta \mu / 2}|F(\mu)|^{2}+\int_{|u|>\Delta u / 2}|f(u)|^{2} d u
\end{aligned}
$$

meaning that

$$
\begin{aligned}
& E \int_{-\infty}^{\infty}\left|f(u)-f_{\sim \mu}, \Delta u(u)\right|^{2} d u \\
& \approx E \int_{|u|>\Delta u / 2}|f(u)|^{2} d u+E \int_{|\mu|>\Delta \mu / 2}|F(\mu)|^{2} d \mu \\
& =\int_{|u|>\Delta u / 2} K_{f}(u, u) d u+\int_{|\mu|>\Delta \mu / 2} S_{f}(\mu, \mu) d \mu
\end{aligned}
$$

holds if the sampling parameters $\Delta u$ and $\Delta \mu$ are sufficiently large. In a similar way, it follows from (16) that the approximation $E \int_{-\infty}^{\infty}\left|f(u)-f_{\Delta u, \Delta \mu}(u)\right|^{2}$ $d u \approx \int_{|u|>\Delta u / 2} K_{f}(u, u) d u+\int_{|\mu|>\Delta \mu / 2} S_{f}(\mu, \mu) d \mu$ is also valid.
In formal terms, (19) can be expressed as

$$
\lim _{\Delta \mu \rightarrow \infty} \frac{\sum_{\lfloor\Delta u \Delta \mu / 2\rfloor<|n|} \frac{1}{\Delta \mu}\left|f_{\Delta \mu}\left(\frac{n}{\Delta \mu}\right)\right|^{2}}{\int_{|u|>\frac{\mid \Delta u \Delta \mu / 2\rfloor}{\Delta \mu}}\left|f_{\Delta \mu}(u)\right|^{2} d u}=1
$$

As $\Delta \mu \rightarrow \infty$, the bandlimited function approaches $f(u)$ :

$$
\lim _{\Delta \mu \rightarrow \infty} \frac{\int_{|u|>\frac{|\Delta u \Delta \mu / 2|}{\Delta \mu}\left|f_{\Delta \mu}(u)\right|^{2} d u}}{\int_{|u|>\Delta u / 2}|f(u)|^{2} d u}=1,
$$

Combining the above two equations leads to

$$
\lim _{\Delta \mu \rightarrow \infty} \frac{\sum_{\lfloor\Delta u \Delta \mu / 2\rfloor<|n|} \frac{1}{\Delta \mu}\left|f_{\Delta \mu}\left(\frac{n}{\Delta \mu}\right)\right|^{2}}{\int_{|u|>\Delta u / 2}|f(u)|^{2} d u}=1
$$

with probability 1 . Then, using (15) we can write that

$$
\lim _{\Delta \mu \rightarrow \infty} \frac{\int_{-\infty}^{\infty}\left|f(u)-f_{\Delta \mu, \Delta u}(u)\right|^{2} d u}{\int_{|u|>\Delta u / 2}|f(u)|^{2} d u+\int_{|\mu|>\Delta \mu / 2}|F(\mu)|^{2} d \mu}=1
$$

holds true with probability 1 . The dual expression

$$
\lim _{\Delta u \rightarrow \infty} \frac{\int_{-\infty}^{\infty}\left|f(u)-f_{\Delta u, \Delta \mu}(u)\right|^{2} d u}{\int_{|u|>\Delta u / 2}|f(u)|^{2} d u+\int_{|\mu|>\Delta \mu / 2}|F(\mu)|^{2} d \mu}=1
$$

follows similarly. These justify the following theorem.

Theorem 2: Let $f: \mathbb{R} \rightarrow \mathbb{C}$ be a random field and let $f_{\Delta \mu, \Delta u}(u)$ be its reconstruction as in (2). Then, the reconstruction error satisfies

$\lim _{\Delta \mu \rightarrow \infty} E\left[\frac{\int_{-\infty}^{\infty}\left|f(u)-f_{\Delta \mu, \Delta u}(u)\right|^{2} d u}{\int_{|u|>\Delta u / 2}|f(u)|^{2} d u+\int_{|\mu|>\Delta \mu / 2}|F(\mu)|^{2} d \mu}\right]=1$

A similar result is valid for the reconstruction $f_{\Delta u, \Delta \mu}(u)$.

It is noteworthy to observe that under reasonabte assumptions, the reconstruction error for the finite-sample representation considered here simplifies to the sum of two terms. These are the energies contained in the truncated "tails" of the function in the spatial domain and the spectral domain. If a function is of finite extent in the frequency domain, the sampling theorem says that finitely spaced samples in the original space domain will be sufficient to reconstruct it. If the function could also be of finite extent in the original space domain, then clearly we would need to retain only those of these finitely-spaced samples falling within the extent of the signal, leading to a finite number of samples that exactly represented the signal. However, it is a fundamental fact that a signal cannot have finite extent in both the space and frequency domains. Therefore, we cannot have a finite number of finitely-spaced samples that exactly represent our signal - the samples would have to be either infinitesimally spaced or extend over an infinite extent, or both, to ensure an exact representation. The same argument can be repeated with the roles of the space and frequency variables interchanged. It follows that we can only have a finite representation if we pretend that the signal has a finite extent in both domains by ignoring, or neglecting, parts of the signal that lie outside those assumed finite extents. The above result shows that the energy of the reconstruction error that results, is given by the energy of 
the parts of the signal that we neglect. In other words, whatever we choose to neglect, returns to us as an error in reconstruction.

Results consistent with this were observed in the numerical simulations in [48], [49], where the authors note that the approximation error for linear canonical transform computation algorithms is basically determined by the error in approximating the continuous Fourier transform by the discrete Fourier transform (DFT), with the error being comparable to the amount of energy falling outside the space and frequency extents implicitly defined by the DFT employed.

According to (21), the reconstruction error is the sum of the energies of the signal left outside the intervals $\Delta u$ and $\Delta \mu$ in the space and frequency domains respectively. Reducing the error requires increasing both $\Delta u$ and $\Delta \mu$. On the other hand, according to (3), the number of samples is given by $\Delta u \Delta \mu$, leading to the expected result that reducing the error requires the acceptance of a greater number of samples, or that reducing the number of samples requires the tolerance of a greater error. But what is the minimum number of samples for a specified error, or the smallest error for a specified number of samples? To answer these questions, one needs to optimize these two parameters in order to minimize the error expression given by (21). This optimization is undertaken in Section V. Before that, for completeness and generality, we will consider the corresponding analysis for the case where anti-aliasing is not used before sampling. The following section can be omitted without loss of continuity.

\section{ERROR ANALYSIS FOR RECONSTRUCTION WITHOUT PRE-FILTERING}

The finite-sample representation of Section II makes use of anti-aliasing filtering before sampling. In this section, we analyze the reconstruction error for the case when an anti-aliasing filter is not used. In the absence of pre-filtering, the finite-sample representation signal takes the form

$$
\hat{f}_{\Delta \mu, \Delta u}(u)=\sum_{n=-\lfloor\Delta u \Delta \mu / 2\rfloor}^{\lfloor\Delta u \Delta \mu / 2\rfloor} f\left(\frac{n}{\Delta \mu}\right) \operatorname{sinc}(\Delta \mu u-n)
$$

having the Fourier transform $\hat{F}_{\Delta \mu, \Delta \sim}$. Let $\hat{f}_{\Delta \mu}$ be defined as

$$
\hat{f}_{\Delta \mu}(u)=\sum_{n=-\infty}^{\infty} f\left(\frac{n}{\Delta \mu}\right) \operatorname{sinc}(\Delta \mu u-n) .
$$

The Fourier transform $\hat{F}_{\Delta \mu}(\mu)$ of $\hat{f}_{\Delta \mu}$ does not agree with $F(\mu)$ on the interval $[-\Delta \mu / 2, \Delta \mu / 2]$ because of aliasing. Hence, we have

$$
\begin{aligned}
& \left\langle F(\mu)-\hat{F}_{\Delta \mu}(\mu), \hat{F}_{\Delta \mu}(\mu)-\hat{F}_{\Delta \mu, \Delta \sim}(\mu)\right\rangle \neq 0 \\
& \int_{-\infty}^{\infty}\left|f(u)-\hat{f}_{\Delta \mu, \Delta u}(u)\right|^{2} d u \neq \int_{-\infty}^{\infty}\left|f(u)-\hat{f}_{\Delta \mu}(u)\right|^{2} d u \\
& \quad+\int_{-\infty}^{\infty}\left|\hat{f}_{\Delta \mu}(u)-\hat{f}_{\Delta \mu, \Delta u}(u)\right|^{2} d u
\end{aligned}
$$

as opposed to the equalities (8) and (9). Nevertheless, the error term $\int\left|f(u)-\hat{f}_{\Delta \mu, \Delta u}(u)\right|^{2} d u$ can be bounded as

$$
\begin{aligned}
& \left(\int_{-\infty}^{\infty}\left|f(u)-\hat{f}_{\Delta \mu} \hat{\sim}_{\Delta u}(u)\right|^{2} d u\right)^{1 / 2} \\
& \leq\left(\int_{-\infty}^{\infty}\left|f(u)-\hat{f}_{\Delta \mu}(u)\right|^{2} d u\right)^{1 / 2} \\
& +\left(\int_{-\infty}^{\infty}\left|\hat{f}_{\Delta \mu}(u)-\hat{f}_{\Delta \mu}{ }_{\sim}(u)\right|^{2} d u\right)^{1 / 2}
\end{aligned}
$$

Then, we obtain from (14) that

$$
\begin{aligned}
\int_{-\infty}^{\infty}\left|\hat{f}_{\Delta \mu}(u)-\hat{f}_{\Delta \mu, \Delta u}(u)\right|^{2} d u & =\sum_{\lfloor\Delta u \mu / 2\rfloor<|n|} \frac{1}{\Delta \mu}\left|f\left(\frac{n}{\Delta \mu}\right)\right|^{2} \\
& \approx \int_{|u|>\Delta u / 2}|f(u)|^{2} d u
\end{aligned}
$$

Inserting (25) in (24), we get

$$
\begin{aligned}
& \left(\int_{-\infty}^{\infty}\left|f(u)-\hat{f}_{\Delta \mu, \Delta u}(u)\right|^{2} d u\right)^{1 / 2} \\
& \leq\left(\int_{-\infty}^{\infty}\left|f(u)-\hat{f}_{\Delta \mu}(u)\right|^{2} d u\right)^{1 / 2}+\left(\int_{|u|>\Delta u / 2}|f(u)|^{2} d u\right)^{1 / 2} \\
& =\left(\int_{-\infty}^{\infty}\left|F(\mu)-\hat{F}_{\Delta \mu}(\mu)\right|^{2} d \mu\right)^{1 / 2}+\left(\int_{|u|>\Delta u / 2}|f(u)|^{2} d u\right)^{1 / 2} .
\end{aligned}
$$

To proceed further with the expression for the error, let us more closely examine the first term appearing in the last line of (26), and write

$$
\begin{aligned}
& \int_{-\infty}^{\infty}\left|F(\mu)-\hat{F}_{\Delta \mu}(\mu)\right|^{2} d \mu=\int_{|\mu|>\Delta \mu / 2}|F(\mu)|^{2} d \mu \\
& \quad+\int_{-\Delta \mu / 2}^{\Delta \mu / 2}\left|F(\mu)-\hat{F}_{\Delta \mu}(\mu)\right|^{2} d \mu \\
& \geq \int_{|\mu|>\Delta \mu / 2}|F(\mu)|^{2} d \mu+\frac{1}{\Delta \mu}\left(\int_{-\Delta \mu / 2}^{\Delta \mu / 2}\left|F(\mu)-\hat{F}_{\Delta \mu}(\mu)\right| d \mu\right)^{2}
\end{aligned}
$$

where (27) is due to the Cauchy-Schwarz inequality. Then, we make use of Nyquist's sampling theorem to express $\hat{F}_{\Delta \mu}$ as

$$
\hat{F}_{\Delta \mu}(\mu)=\operatorname{rect}\left(\frac{\mu}{\Delta \mu}\right) \sum_{n=-\infty}^{\infty} F(\mu-\Delta \mu n)
$$


under the assumption that $\int_{-\infty}^{\infty}|F(\mu)| d \mu<\infty$. Then, it follows from (28) that

$$
\begin{aligned}
& \int_{-\Delta \mu / 2}^{\Delta \mu / 2}\left|F(\mu)-\hat{F}_{\Delta \mu}(\mu)\right| d \mu \\
& =\int_{-\Delta \mu / 2}^{\Delta \mu / 2}\left|\sum_{n \neq 0} F(\mu-\Delta \mu n)\right| d \mu \\
& \leq \int_{-\Delta \mu / 2}^{\Delta \mu / 2} \sum_{n \neq 0}|F(\mu-\Delta \mu n)| d \mu .
\end{aligned}
$$

Noting that

$$
\begin{aligned}
& \sum_{n=-k}^{k} \int_{-\Delta \mu / 2}^{\Delta \mu / 2}|F(\mu-\Delta \mu n)| d \mu \\
& \quad=\int_{\mu=-\frac{k+1}{2} \Delta \mu}^{\frac{k+1}{2} \Delta \mu}|F(\mu)| d \mu-\int_{-\Delta \mu / 2}^{\Delta \mu / 2}|F(\mu)| d \mu \\
& \quad \leq \int_{|\mu|>\Delta \mu / 2}|F(\mu)| d \mu<\infty
\end{aligned}
$$

holds true for all $k \in \mathbb{N}$, we conclude

$$
\int_{-\Delta \mu / 2}^{\Delta \mu / 2} \sum_{n \neq 0}|F(\mu-\Delta \mu n)| d \mu=\int_{|\mu|>\Delta \mu / 2}|F(\mu)| d \mu
$$

from the dominated convergence theorem.

The inequality (27) and the equality (31) suggest that the aliasing error $\int_{-\infty}^{\infty}\left|F(\mu)-\hat{F}_{\Delta \mu}(\mu)\right|^{2} d \mu$ is proportional to $\int_{|\mu|>\Delta \mu / 2}|F(\mu)| d \mu$. Combining this observation with (26), we see that it is the 1-norm integral $\int_{|\mu|>\Delta \mu / 2}|F(\mu)| d \mu$ which dominates the upper bound of the reconstruction error given by (26). This is also consistent with the theorem proved in [50] which states that $\left|f(u)-\hat{f}_{\Delta \mu, \Delta u}(u)\right| \leq 2 \int_{|\mu|>\Delta \mu / 2}|F(\mu)| d \mu$ is valid for all $u \in \mathbb{R}$. There are counter-examples in the literature [7] showing that $\int_{-\infty}^{\infty}\left|F(\mu)-\hat{F}_{\Delta \mu}(\mu)\right|^{2} d \mu$ cannot be bounded above by a universal constant $C$ times the 2-norm integral $\int_{|\mu|>\Delta \mu / 2}|F(\mu)|^{2} d \mu$. Both the analysis we have carried out here and previous results appearing in [7], [50] indicate that the use of anti-alias filtering is helpful in reducing the reconstruction error. Since it also makes the analysis more transparent and simpler, we continue with that choice.

\section{OPTIMIZATION OF THE EXTENTS VIA LAGRANGE MULTIPLIERS}

Returning to where we left at the end of Section II, we had found that both the number of samples $n(\Delta u, \Delta \mu)$ and the reconstruction error $e(\Delta u, \Delta \mu)$ were functions of the extents $\Delta u$ and $\Delta \mu$, as given by (3) and (21):

$$
\begin{aligned}
n(\Delta u, \Delta \mu) & =\Delta u \Delta \mu \\
e(\Delta u, \Delta \mu) & =\int_{|u|>\Delta u / 2} K_{f}(u, u) d u+\int_{|\mu|>\Delta \mu / 2} S_{f}(\mu, \mu) d \mu
\end{aligned}
$$

Here the reconstruction error can be considered to be a performance parameter, while the number of samples can be considered to be a cost parameter. The extents $\Delta u$ and $\Delta \mu$ are intermediate parameters that we are free to choose but which are not of interest in themselves. It is clear that greater performan- ce requires greater cost, and that lower cost requires acceptance of lower performance. So we can pose two fundamental problems: (i) Find the minimum number of samples $n(\Delta u, \Delta \mu)$ to achieve a specified reconstruction error $e(\Delta u, \Delta \mu)$; and, (ii) Find the minimum error $e(\Delta u, \Delta \mu)$ for a given number of samples $n(\Delta u, \Delta \mu)$. Actually, both problems are related and their solution constitutes the Pareto-optimal tradeoff between the sample count and the error, obtained after the parameters $\Delta u$ and $\Delta \mu$ have been optimized out. The following theorem characterizes the Pareto optimal $(\Delta u, \Delta \mu)$ pairs.

Theorem 3: Let $f: \mathbb{R} \rightarrow \mathbb{C}$ be a random field and let $f_{\Delta \mu, \Delta u}(u)$ be its reconstruction as in (2). Then, the Pareto optimal $(\Delta u, \Delta \mu)$ pairs satisfy the equation

$$
\frac{\Delta \mu}{\Delta u}=\frac{K_{f}\left(\frac{\Delta u}{2}, \frac{\Delta u}{2}\right)+K_{f}\left(-\frac{\Delta u}{2},-\frac{\Delta u}{2}\right)}{S_{f}\left(\frac{\Delta \mu}{2}, \frac{\Delta \mu}{2}\right)+S_{f}\left(-\frac{\Delta \mu}{2},-\frac{\Delta \mu}{2}\right)} .
$$

Proof: The Lagrange multipliers method implies the existence of a $\lambda \in \mathbb{R}$ such that the optimal $(\Delta u, \Delta \mu)$ pair satisfies

$$
\frac{\partial e(\Delta u, \Delta \mu)}{\partial \Delta u}+\lambda \Delta \mu=0, \frac{\partial e(\Delta u, \Delta \mu)}{\partial \Delta \mu}+\lambda \Delta u=0
$$

Note that $e(\Delta u, \Delta \mu)$ can be expressed as $e(\Delta u, \Delta \mu)=$ $e_{1}(\Delta u)+e_{2}(\Delta \mu)$, where

$$
\begin{aligned}
e_{1}(x) & =E_{0}-\int_{-x / 2}^{x / 2} K_{f}\left(x^{\prime}, x^{\prime}\right) d x^{\prime} \\
e_{2}(y) & =E_{0}-\int_{-y / 2}^{y / 2} S_{f}\left(y^{\prime}, y^{\prime}\right) d y^{\prime} \\
E_{0} & =\int_{-\infty}^{\infty} K_{f}\left(x^{\prime}, x^{\prime}\right) d x^{\prime}=\int_{-\infty}^{\infty} S_{f}\left(y^{\prime}, y^{\prime}\right) d y^{\prime}
\end{aligned}
$$

Rewriting (35) as

$$
e_{1}^{\prime}(\Delta u)+\lambda \Delta \mu=0, \quad e_{2}^{\prime}(\Delta \mu)+\lambda \Delta u=0
$$

we get the equality $e_{1}^{\prime}(\Delta u) \Delta u=e_{2}^{\prime}(\Delta \mu) \Delta \mu$, giving rise to Theorem 3.

In order to find the optimal $(\Delta u, \Delta \mu)$ pair, (34) and the constraint equation need to be solved together. The constraint equation is either that $n(\Delta u, \Delta \mu)$ is a given constant, or that $e(\Delta u, \Delta \mu)$ is a given constant; corresponding to the problems of finding the smallest error for given number of samples, or finding the smallest number of samples for given error, respectively. Finally, we can plot the number of samples versus reconstruction error curve consisting of the best achievable (Pareto-optimal) points.

\section{NUMERICAL RESULtS}

We now provide some numerical examples illustrating the relationship between the number of samples and reconstruction error. Our first example is a random field having autocorrelation function

$$
K_{f}\left(u_{1}, u_{2}\right)=\psi_{n}\left(u_{1}\right) \psi_{n}\left(u_{2}\right)
$$

where $\psi_{n}(u)$ is the $\mathrm{n}^{\text {th }}$ order Hermite-Gaussian function. Since Hermite-Gaussian functions are the eigenfunctions of the Fourier transform having eigenvalues of unit magnitude [51], the autocorrelation of the original function and the autocorrelation of its Fourier transform are exactly the same; that is, $K_{f}\left(u_{1}, u_{2}\right)=S_{f}\left(u_{1}, u_{2}\right)$ holds true. Therefore (34) reduces to 


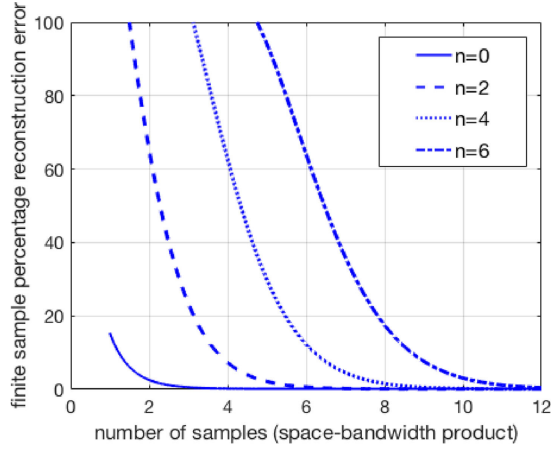

Fig. 1. Pareto-optimal tradeoff curves between finite sample reconstruction error versus number of samples, for the random processes having autocorrelation function $K_{f}\left(u_{1}, u_{2}\right)=\psi_{n}\left(u_{1}\right) \psi_{n}\left(u_{2}\right)$, where $\psi_{n}(u)$ is the $\mathrm{n}^{\text {th }}$ order Hermite-Gaussian function.

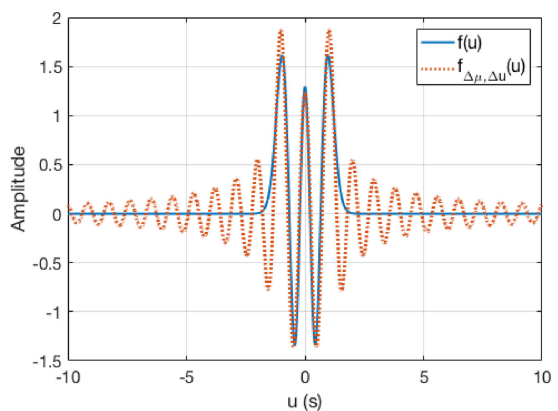

Fig. 2. The random field having an autocorrelation function of the form (36) and its reconstruction for $n=4$ and $N=5$.

$\Delta u=\Delta \mu \times 1 s^{2}$, where $s$ refers to the unit of $\Delta u$ (such as $s=$ 1 meter or $s=1$ second). Then, under the constraint that the number of samples to be used is $N$, the error term $e(\Delta u, \Delta \mu)$ given by (33) takes the form

$$
2 \int_{|u|>\sqrt{N} / 2} \psi_{n}^{2}(u) d u \text {. }
$$

We determined the Pareto-optimal curves $n(\Delta u, \Delta \mu)$ versus $e(\Delta u, \Delta \mu)$ for several values of $n$ using (37), and plotted them in Figure 1.

As the order of the Hermite polynomial increases, both the spatial and the spectral extent of the corresponding HermiteGaussian function increases as well. Therefore, in Figure 1, it is natural to observe that larger $n$ results in usage of more samples to achieve the same error performance. For $n=4$ and $N=5$, a realization of the random field $f(u)$ and its reconstruction $f_{\Delta \mu, \Delta u}(u)$ is given in Figure 2.

Our next example is a random field $f(u)$ having a Gaussian Schell-model (GSM) type autocorrelation function

$$
K_{f}\left(u_{1}, u_{2}\right)=A e^{-\left(u_{1}^{2}+u_{2}^{2}\right) / 4 \sigma_{I}^{2}} e^{-\left(u_{1}-u_{2}\right)^{2} / 2 \sigma_{\mu}^{2}}
$$

This is a parametric non-stationary autocorrelation model widely used in wave propagation problems [52]-[56]. In [52], it is proven that (38) can be decomposed as

$$
K_{f}\left(u_{1}, u_{2}\right)=\sum_{n=-\infty}^{\infty} \lambda_{n} \sqrt{\frac{c}{\pi}} \psi_{n}\left(\sqrt{\frac{c}{\pi}} u_{1}\right) \psi_{n}\left(\sqrt{\frac{c}{\pi}} u_{2}\right),
$$

where $\psi_{n}(u)$ is the $\mathrm{n}^{\text {th }}$ order Hermite-Gaussian function, $\lambda_{n}$ is a positive number depending on $A, \sigma_{I}, \sigma_{\mu}$ and $n$, according to a formula which is explicitly given in [52], and

$$
c=\left(\left(\frac{1}{4 \sigma_{I}^{2}}\right)^{2}+\frac{1}{4 \sigma_{I}^{2} \sigma_{\mu}^{2}}\right)^{1 / 2} .
$$

Now, using the fact that the functions $\psi_{n}(u)$ are the eigenfunctions of the Fourier transform all having unit-magnitude eigenvalues, and using the scaling property of the Fourier transform, we get

$$
\begin{aligned}
S_{f}\left(\mu_{1}, \mu_{2}\right) & =\sum_{n=-\infty}^{\infty} \lambda_{n} \sqrt{\frac{\pi}{c}} \psi_{n}\left(\sqrt{\frac{\pi}{c}} \mu_{1}\right) \psi_{n}\left(\sqrt{\frac{\pi}{c}} \mu_{2}\right) \\
& =\frac{\pi}{c} K_{f}\left(\frac{\pi}{c} \mu_{1}, \frac{\pi}{c} \mu_{2}\right)
\end{aligned}
$$

We insert (40) in (34) to find the relationship between the optimal $\Delta u$ and $\Delta \mu$, leading us to

$$
\frac{K_{f}\left(\frac{\Delta u}{2}, \frac{\Delta u}{2}\right)+K_{f}\left(-\frac{\Delta u}{2},-\frac{\Delta u}{2}\right)}{K_{f}\left(\frac{\pi \Delta \mu}{2 c}, \frac{\pi \Delta \mu}{2 c}\right)+K_{f}\left(-\frac{\pi \Delta \mu}{2 c},-\frac{\pi \Delta \mu}{2 c}\right)}=\frac{c \Delta u}{\pi \Delta \mu} .
$$

from which we see that the optimal $(\Delta u, \Delta \mu)$ pair for a GSM-type source should satisfy $\Delta \mu=\Delta u c / \pi$. Hence, under the constraint $\Delta u \Delta \mu=N$, we find the optimal pair to be $(\Delta u, \Delta \mu)=(\sqrt{N \pi / c}, \sqrt{N c / \pi})$. In this case, the best achievable error is given by

$$
\begin{aligned}
& e(\Delta u, \Delta \mu) \\
& =\int_{|u|>\Delta u / 2} K_{f}(u, u) d u+\int_{|\mu|>\Delta u c / 2 \pi} \frac{\pi}{c} K_{f}\left(\frac{\pi}{c} \mu, \frac{\pi}{c} \mu\right) d \mu \\
& =2 \int_{|u|>\Delta u / 2} K_{f}(u, u) d u=2 A^{2} \int_{|u|>\sqrt{N \pi / 4 c}} e^{-u^{2} / 2 \sigma_{I}^{2}} d u \\
& =4 A^{2} \sqrt{2 \pi} \sigma_{I} Q\left(\sqrt{\frac{N \pi}{4 c \sigma_{I}^{2}}}\right)
\end{aligned}
$$

where $Q(x)=\frac{1}{\sqrt{2 \pi}} \int_{x}^{\infty} e^{-x^{2} / 2} d x$ refers to the Q-function.

Now, let us express this minimum achievable average error in relative form, by normalizing it by the average energy of $f(u)$ as follows:

$$
\begin{aligned}
\frac{e(\Delta u, \Delta \mu)}{\int_{-\infty}^{\infty} K_{f}(u, u) d u} & =\frac{e(\Delta u, \Delta \mu)}{\int_{-\infty}^{\infty} A^{2} e^{-u^{2} / 2 \sigma_{I}^{2}} d u} \\
& =\frac{e(\Delta u, \Delta \mu)}{A^{2} \sqrt{2 \pi} \sigma_{I}}=4 Q\left(\sqrt{\frac{N \pi}{4 c \sigma_{I}^{2}}}\right)
\end{aligned}
$$

Setting the insignificant amplitude factor $A$ aside, the two parameters that determine a GSM-type source are $\sigma_{I}$ and $\sigma_{\mu}$. If both of these two parameters are increased $\kappa$ times, then $c$ decreases $\kappa^{2}$ times. Therefore $c \sigma_{I}^{2}$ does not change. This in turn means that the above normalized error stays the same as well. Hence, we conclude that the normalized best achievable error depends only on the ratio $\sigma_{I} / \sigma_{\mu}$. Thus in our numerical examples we do not have to vary $\sigma_{I}$ and $\sigma_{\mu}$ independently; we need only consider different ratios of them.

Figure 3 illustrates Pareto-optimal tradeoff curves for normalized error versus number of samples, for different values of $\sigma_{I} / \sigma_{\mu}$. The normalized error given in (43) has been expressed as a percentage by multiplying with 100 . As the intensity width $\sigma_{I}$ increases and the correlation width $\sigma_{\mu}$ decreases, the number of 


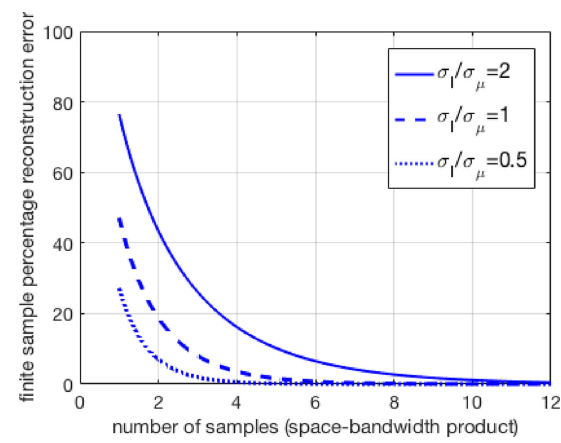

Fig. 3. Pareto-optimal tradeoff curves between finite sample reconstruction error versus number of samples, for GSM-type sources.

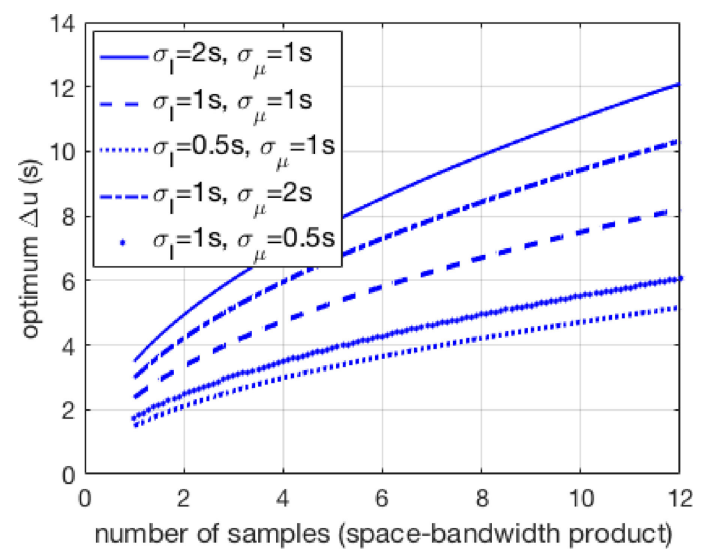

Fig. 4. Optimum value of $\Delta u$ versus the number of samples, for GSM-type source.

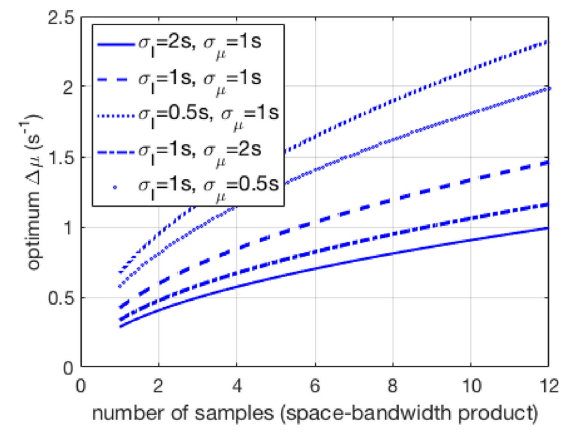

Fig. 5. Optimum value of $\Delta \mu$ versus the number of samples, for GSM-type source.

independent samples having non-negligible variance increases. Therefore, it is natural to observe that higher $\sigma_{I} / \sigma_{\mu}$ ratios result in the usage of more samples to achieve the same error.

The optimum values of $\Delta u=\sqrt{N \pi / c}$ and $\Delta \mu=\sqrt{N c / \pi}$ as a function of the number of samples $N$ are shown in Figure 4 and Figure 5, respectively. We conclude from these figures that the optimum value of $\Delta u$ increases as $\sigma_{I}$ or $\sigma_{\mu}$ increases, whereas the optimum value of $\Delta \mu$ is inversely proportional to $\sigma_{I}$ and $\sigma_{\mu}$. We also see from Figure 4 and Figure 5 that the $\left(\sigma_{I}, \sigma_{\mu}\right)$ pair having the largest optimal $\Delta u$ has the smallest optimal $\Delta \mu$, and vice versa.

Moreover, comparing the curve of the $\left(\sigma_{I}, \sigma_{\mu}\right)$ pair $(1 s, 0.5 s)$ with that of $(2 s, 1 s)$, or comparing the curve of $(0.5 s, 1 s)$ with that of $(1 s, 2 s)$, we can verify that if both $\sigma_{I}$ and $\sigma_{\mu}$ are increased by the same factor $\kappa$, then $c$ decreases by the factor $\kappa^{2}$,

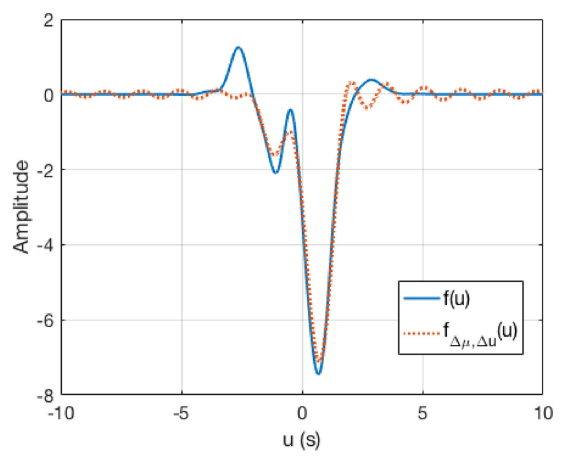

Fig. 6. The random field having an autocorrelation function of the form (38) and its reconstruction for $\sigma_{I}=1, \sigma_{\mu}=0.5$ and $N=5$.

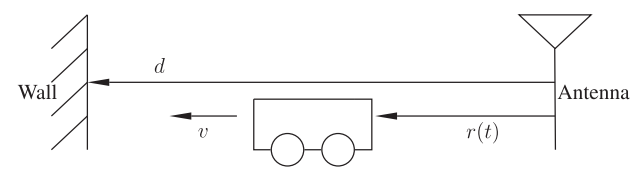

Fig. 7. Transmitting antenna with reflecting wall. After [57].

resulting in a $\kappa$-times increase in the optimum $\Delta u$ and a $\kappa$-times decrease in the optimum $\Delta \mu$. For $\sigma_{I}=1, \sigma_{\mu}=0.5$ and $N=5$, a realization of the random field $f(u)$ and its reconstruction $f_{\Delta \mu, \Delta u}(u)$ is given in Figure 6.

Our final example is based on a wireless communications channel model. Specifically, we consider the fading multipath channel given in [57]: $h(t, \tau)=\sum_{i} a_{i}(t) \delta\left(t-\tau-\tau_{i}(t)\right)$ is the response at time $t$ to an impulse generated at time $\tau$. Here $a_{i}(t)$ and $\tau_{i}(t)$ respectively denote the attenuation and propagation delay at time $t$ from the transmitter to the receiver on path $i$. For a receiver having bandwidth $W$, the impulse response takes the form

$$
h(t, \tau)=\sum_{i} a_{i}(t) W \operatorname{sinc}\left(W\left(t-\tau-\tau_{i}(t)\right)\right) .
$$

If unit power white noise is applied to this channel as the input, we get a random field at the output having autocorrelation function

$$
\begin{aligned}
K_{f}\left(t_{1}, t_{2}\right) & =\int_{-\infty}^{\infty} h\left(t_{1}, \tau\right) h^{*}\left(t_{2}, \tau\right) d \tau=\sum_{i} \sum_{j} a_{i}\left(t_{1}\right) a_{j}\left(t_{2}\right) \\
& \times W \operatorname{sinc}\left(W\left(t_{1}-t_{2}-\left(\tau_{i}\left(t_{1}\right)-\tau_{j}\left(t_{2}\right)\right)\right)\right)
\end{aligned}
$$

For the example of a perfectly reflecting wall shown in Figure 7, the attenuation and propagation delay terms can be expressed as [57]

$$
\begin{array}{ll}
a_{1}(t)=\frac{|\alpha|}{r_{0}+v t}, & a_{2}(t)=\frac{|\alpha|}{2 d-r_{0}-v t}, \\
\tau_{1}(t)=\frac{r_{0}+v t}{c}, & \tau_{2}(t)=\frac{2 d-r_{0}-v t}{c},
\end{array}
$$

where $c$ is the speed of light, and the effect of phase change at the reflector on $\tau_{2}(t)$ is neglected.

Inserting (46) in (45) with the parameters $\alpha=1, r_{0}=$ $1.5 \mathrm{~km}, d=2 \mathrm{~km}, v=100 \mathrm{~km} / \mathrm{h}, W=50 \mathrm{~Hz}$, and $0 \leq t_{1}$, $t_{2} \leq 10 \mathrm{sec}$, we get an autocorrelation function for which the performance curves are provided in Figure 8 and Figure 9. For $\mathrm{N}=400$, a realization of the random field $f(u)$ and its reconstruction $f_{\Delta \mu, \Delta u}(u)$ is given in Figure 10. The different 


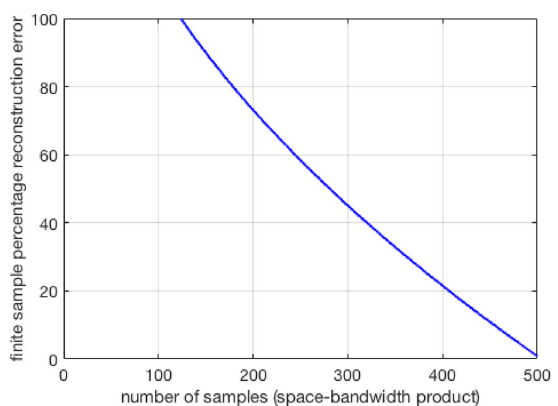

Fig. 8. Pareto-optimal tradeoff curves between finite sample reconstruction error versus number of samples, for the fading multipath channel output.

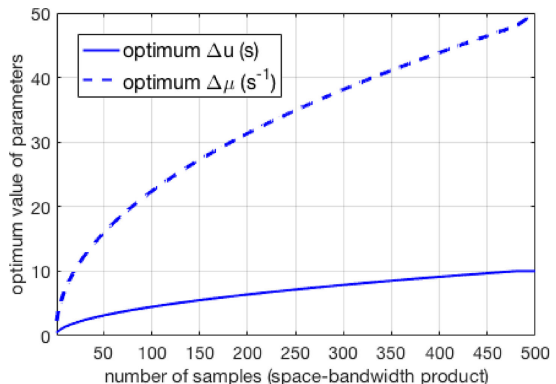

Fig. 9. Optimum values of $\Delta u$ and $\Delta \mu$, versus the number of samples, for the fading multipath channel output.

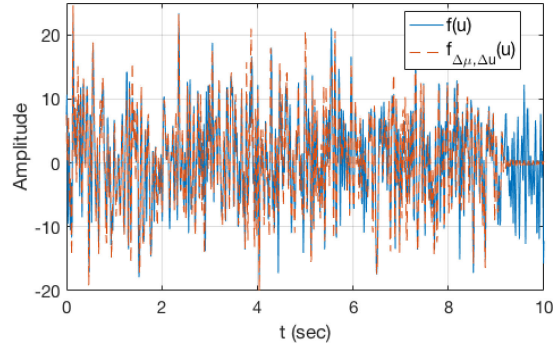

Fig. 10. The random field having an autocorrelation function of the form (45) and its reconstruction for $N=400$.

TABLE I

RECONSTRUCTION ERROR FOR DIFFERENT VALUES OF $\Delta u$ AND $\Delta \mu$. THE OPTIMAL PAIR IS SHOWN IN BOLD

\begin{tabular}{|l|l|c|}
\hline$\Delta u$ & $\Delta \mu$ & Reconstruction error \\
\hline 8 & 50 & $35.29 \%$ \\
\hline 9 & 44.44 & $31.14 \%$ \\
\hline $\mathbf{9 . 1 2}$ & $\mathbf{4 3 . 8 6}$ & $\mathbf{3 1 . 0 7} \%$ \\
\hline 9.5 & 42.11 & $34.53 \%$ \\
\hline 10 & 40 & $37.13 \%$ \\
\hline
\end{tabular}

values that the reconstruction error takes for different values of $\Delta u$ and $\Delta \mu$ under the constraint $N=400$ is given in Table I. The experimental results verify the $(\Delta u, \Delta \mu)$ pair found using Theorem 3 is optimal.

These examples show us the least error for a given number of samples, or the least number of samples for a given error, and also tell us how to sample in order to ensure we are on an optimal tradeoff point.

\section{CONCLUSION}

We addressed the problem of representing a non-stationary finite-energy random field using finitely many samples. In order to have finitely many samples, the extent over which sampling takes place, as well as the spacing between the samples must be finite, the latter which depends on a finite extent in the frequency domain. While a signal cannot have finite extent in both the space and frequency domains to begin with, we allow it to have infinite extent in both domains for generality. Therefore, it becomes necessary to neglect the content of the signal outside assumed extents in both domains, creating a source of reconstruction error. Based on the proposed formulation, we proved that this reconstruction error can be made arbitrarily small by choosing the number of samples large enough. More interestingly, we showed that the error is approximately the sum of the energies falling outside the assumed extents in the spatial and spectral domains; that is, the reconstruction error is equal to the energy of the truncated parts of the signal. Whatever we neglect at the beginning, returns to us as an error at the end.

The spatial extent $\Delta u$ and spectral extent $\Delta \mu$ are the two key parameters defining the representation, which determine both the reconstruction error and the total number of samples. By optimizing these parameters we are able to find the tightest (Pareto-optimal) tradeoff curves between the sample count and the error. We can think of error as performance and number of samples as cost. Thus, in a given application these curves would allow us to choose the best possible performance for given cost, or the lowest possible cost for given performance.

Since we considered the sampling problem in its most basic form and obtained analytical results, we have been able to transparently and precisely portray what is involved in representing non-stationary finite-energy signals with finitely many samples. The tradeoff between reconstruction error and sample count, its dependence on how we choose the sampling parameters, and how we can optimize this tradeoff, have been made explicit. The only information needed to produce this tradeoff curve is the autocorrelation. Thus if we know or can estimate or model the autocorrelation for a family of signals, then we can determine the optimal sampling strategy (the optimal operating point on the Pareto-optimal tradeoff curve) based on the relative importance of error and sample count in a given practical situation.

Our approach also amounts to bounding the number of degrees of freedom of a field whose autocorrelation function is given. We have seen that this is the minimum number of samples with which the signal can be represented and recovered, and that it depends on the specified error tolerance and is not an absolute concept.

\section{REFERENCES}

[1] A. J. Jerri, "The Shannon sampling theorem-Its various extensions and applications: A tutorial review," Proc. IEEE, vol. 65, no. 11, pp. 15651596, Nov. 1977.

[2] P. L. Butzer and R. L. Stens, "Sampling theory for not necessarily bandlimited functions: A historical overview," SIAM Rev., vol. 34, no. 1, pp. 40-53, 1992.

[3] M. Unser, "Sampling-50 years after Shannon," Proc. IEEE, vol. 88, no. 4, pp. 569-587, Apr. 2000.

[4] P. P. Vaidyanathan, "Generalizations of the sampling theorem: Seven decades after Nyquist," IEEE Trans. Circuits Syst. I, Fundam. Theory Appl., vol. 48, no. 9, pp. 1094-1109, Sep. 2001.

[5] M. Zakai, "Band-limited functions and the sampling theorem," Inf. Comput./Inf. Control, vol. 8, no. 2, pp. 143-158, 1965.

[6] P. J. S. G. Ferreira, "On the approximation of nonbandlimited signals by nonuniform sampling series," in Proc. Eur. Signal Process. Conf., 1996, pp. $1567-1570$. 
[7] J. L. Brown, "Estimation of energy aliasing error for nonbandlimited signals," Multdimensional Syst. Signal Process., vol. 15, no. 1, pp. 51-56, 2004.

[8] J. L. Brown, "Bounds for truncation error in sampling expansions of bandlimited signals," IEEE Trans. Inf. Theory, vol. IT-15, no. 4, pp. 440-444, 1969.

[9] H. S. Piper, "Best asymptotic bounds for truncation error in sampling expansions of band-limited signals (Corresp.)," IEEE Trans. Inf. Theory, vol. IT-21, no. 6, pp. 687-690, Nov. 1975.

[10] S. Cambanis and E. Masry, "Truncation error bounds for the cardinal sampling expansion of band-limited signals," IEEE Trans. Inf. Theory, vol. IT-28, no. 4, pp. 605-612, Jul. 1982.

[11] F. Krahmer and R. Ward, "Stable and robust sampling strategies for compressive imaging," IEEE Trans. Image Process., vol. IP-23, no. 2, pp. 612-622, Feb. 2014.

[12] H. G. Feichtinger and K. Grochenig, "Irregular sampling theorems and series expansions of band-limited functions," J. Math. Anal. Appl., vol. 167 , no. 2, pp. 530-556, 1992.

[13] K. Grochenig, "Reconstruction algorithms in irregular sampling," Math. Comput., vol. 59, no. 199, pp. 181-194, 1992.

[14] A. Aldroubi and H. Feichtinger, "Exact iterative reconstruction algorithm for multivariate irregularly sampled functions in spline-like spaces: The $L_{p}$ theory," Proc. Amer. Math. Soc., vol. 126, no. 9, pp. 2677-2686, 1998.

[15] W. Chen, S. Itoh, and J. Shiki, "Irregular sampling theorems for wavelet subspaces," IEEE Trans. Inf. Theory, vol. 44, no. 3, pp. 1131-1142, May 1998.

[16] A. Özçelikkale, H. M. Ozaktas, and E. Arıkan, "Signal recovery with cost constrained measurements," IEEE Trans. Signal Process., vol. 58, no. 7, pp. 3607-3617, Jul.2010.

[17] A. Özçelikkale and H. M. Ozaktas, "Representation of optical fields using finite numbers of bits," Opt. Lett., vol. 37, pp. 2193-2195, 2012.

[18] A. Özçelikkale and H. M. Ozaktas, "Beyond Nyquist sampling: A costbased approach," J. Opt. Soc. Amer. A, vol. 30, no. 4, pp. 645-655, 2013.

[19] A. Özçelikkale and H. Ozaktas, "Optimal representation of non-stationary random fields with finite numbers of samples: A linear MMSE framework," Digit. Signal Process., vol. 23, no. 5, pp. 1602-1609, 2013.

[20] M. Vetterli and C. Herley, "Wavelets and filter banks-theory and design," IEEE Trans. Signal Process., vol. 40, no. 9, pp. 2207-2232, Sep. 1992.

[21] M. Unser, A. Aldroubi, and M. Eden, "On the asymptotic convergence of B-spline wavelets to Gabor functions," IEEE Trans. Inf. Theory, vol. 38, no. 2, pp. 864-872, Mar. 1992.

[22] M. Unser and A. Aldroubi, "Polynomial splines and wavelets-A signal processing perspective," in Wavelets-A Tutorial in Theory and Applications. New York, NY, USA: Academic, 1992, pp. 91-122.

[23] H. A. Inan and A. T. Erdogan, "A convolutive bounded component analysis framework for potentially nonstationary independent and/or dependent sources," IEEE Trans. Signal. Process., vol. 63, no. 1, pp. 18-30, Jan. 2015.

[24] Y. Liu, O. Kosut, and A. S. Willsky, "Sampling from Gaussian Markov random fields using stationary and non-stationary subgraph perturbations," IEEE Trans. Signal Process., vol. 63, no. 3, pp. 576-589, Feb. 2015.

[25] Y. Yang, X. Dong, Z. Peng, W. Zhang, and G. Meng, "Component extraction for non-stationary multi-component signal using parameterized de-chirping and band-pass filter," IEEE Signal Process. Lett., vol. 22, no. 9 , pp. 1373-1377, Sep. 2015.

[26] R. Tavares and R. Coelho, "Speech enhancement with nonstationary acoustic noise detection in time domain," IEEE Signal Process. Lett., vol. 23, no. 1, pp. 6-10, Jan. 2016.

[27] S. M. Nørholm, J. R. Jensen, and M. G. Christensen, "Enhancement and noise statistics estimation for non-stationary voiced speech," IEEE/ACM Trans. Audio, Speech, Lang. Process., vol. 24, no. 4, pp. 645-658, Apr. 2016.

[28] P. Flandrin, A. Napolitano, H. M. Ozaktas, and D. J. Thomson, "Recent advances in theory and methods for nonstationary signal analysis (editorial)," EURASIP J. Adv. Signal Process., vol. 2011, 2011, Art. no. 963642.

[29] W. A. Gardner, "A sampling theorem for nonstationary random processes," IEEE Trans. Inf. Theory, vol. IT-18, no. 6, pp. 808-809, Nov. 1972.

[30] F. Garcia, I. Lourtie, and J. Buescu, " $L_{2}(R)$ nonstationary processes and the sampling theorem," IEEE Signal Process. Lett., vol. 8, no. 4, pp. 117-119, Apr. 2001.

[31] A. Napolitano, "Sampling of spectrally correlated processes," IEEE Trans. Signal Process., vol. 59, no. 2, pp. 525-539, Feb. 2011.

[32] F. Gori and G. Guattari, "Shannon number and degrees of freedom of an image," Opt. Commun., vol. 7, no. 2, pp. 163-165, 1973.
[33] A. Starikov, "Effective number of degrees of freedom of partially coherent sources," J. Opt. Soc. Amer., vol. 72, no. 11, pp. 1538-1544, 1982.

[34] D. Mendlovic and A. W. Lohmann, "Space bandwidth product adaptation and its application to superresolution: Fundamentals," J. Opt. Soc. Amer. A, vol. 14 , no. 3, pp. 558-562, 1997.

[35] H. M. Ozaktas and F. S. Oktem, "Phase-space window and degrees of freedom of optical systems with multiple apertures," J. Opt. Soc. Amer. A, vol. 30, no. 4, pp. 682-690, 2013.

[36] R. Piestun and D. A. B. Miller, "Electromagnetic degrees of freedom of an optical system," J. Opt. Soc. Amer. A, vol. 17, no. 5, pp. 892-902, 2000

[37] A. Poon, R. Brodersen, and D. Tse, "Degrees of freedom in multipleantenna channels: a signal space approach," IEEE Trans. Inf. Theory, vol. 51, no. 2, pp. 523-536, Feb. 2005.

[38] M. Migliore, "On the role of the number of degrees of freedom of the field in MIMO channels," IEEE Trans. Antennas Propag., vol. 54, no. 2 , pp. 620-628, Feb. 2006.

[39] C. Wang, T. Gou, and S. A. Jafar, "Subspace alignment chains and the degrees of freedom of the three-user MIMO interference channel," IEEE Trans. Inf. Theory, vol. 60, no. 5, pp. 2432-2479, May 2014.

[40] H.-J. Chou, R. Y. Chang, and J.-M. Wu, "Degrees-of-freedom-based sequential base-station selection in cochannel MIMO networks," IEEE Trans. Veh. Tech., vol. 65, no. 2, pp. 953-959, Feb. 2016.

[41] J. Xie and S. Ulukus, "Secure degrees of freedom of one-hop wireless networks," IEEE Trans. Inf. Theory, vol. 60, no. 6, pp. 3359-3378, 2014.

[42] F. S. Oktem and H. M. Ozaktas, "Exact relation between continuous and discrete linear canonical transforms," IEEE Signal Proc. Lett., vol. 16, no. 8, pp. 727-730, Aug. 2009.

[43] F. S. Oktem and H. M. Ozaktas, "Equivalence of linear canonical transform domains to fractional Fourier domains and the bicanonical width product: A generalization of the space-bandwidth product," J. Opt. Soc. Amer. A, vol. 27 , no. 8 , pp. 1885-1895, 2010.

[44] T. Berger, Rate Distortion Theory: A Mathematical Basis for Data Compression. Englewood Cliffs, NJ, USA: Prentice-Hall, 1971.

[45] N. S. Jayant and P. Noll, Digital Coding of Waveforms: Principles and Applications to Speech and Video. Englewood Cliffs, NJ, USA: PrenticeHall, 1984

[46] J. J. Benedetto and W. Czaja, Integration and Modern Analysis. Cambridge, MA, USA: Birkhäuser.

[47] L. Koralov and Y. Sinai, Theory of Probability and Random Variables, 2nd ed. Berlin, Germany: Springer.

[48] H. M. Ozaktas, A. Koc, I. Sari, and M. A. Kutay, "Efficient computation of quadratic-phase integrals in optics," Opt. Lett., vol. 31, no. 1, pp. 35-37, 2006.

[49] A. Koc, H. M. Ozaktas, C. Candan, and M. A. Kutay, "Digital computation of linear canonical transforms," IEEE Trans. Signal Process., vol. 56, no. 6, pp. 2383-2394, Jun. 2008.

[50] J. L. Brown, Jr., "On the error in reconstructing a non-bandlimited function by means of the bandpass sampling theory," J. Math. Anal. Appl. vol. 18, no. 1 , pp. 75-84, 1967.

[51] H. M. Ozaktas, Z. Zalevsky, and M. A. Kutay, The Fractional Fourier Transform With Applications in Optics and Signal Processing. Hoboken, NJ, USA: Wiley, 2000.

[52] A. Starikov and E. Wolf, "Coherent-mode representation of Gaussian Schell-model sources and of their radiation fields," J. Opt. Soc. Amer. vol. 72, no. 7, pp. 923-928, 1982 .

[53] A. T. Friberg and J. Turunen, "Imaging of Gaussian-Schell mode sources," J. Opt. Soc. Amer. A, vol. 5, no. 5, pp. 713-720, 1988.

[54] Q. Lin and Y. Cai, "Fractional Fourier transform for partially coherent Gaussian-Schell model beams," Opt. Lett., vol. 27, no. 19, pp. 1672 1674, 2002.

[55] S. Zhu, Y. Cai, and O. Korotkova, "Propagation factor of a stochastic electromagnetic Gaussian Schell-model beam," Opt. Express, vol. 18 no. 12, pp. 12587-12598, 2010.

[56] L. Mandel and E. Wolf, Optical Coherence and Quantum Optics. Cambridge, U.K.: Cambridge Univ. Press, 1995.

[57] D. Tse and P. Viswanath, Fundamentals of Wireless Communications. Cambridge, U.K.: Cambridge Univ. Press, 2005.

Talha Cihad Gulcu (S'13), photograph and biography not available at the time of publication.

Haldun M. Ozaktas (M'07-F'13), photograph and biography not available at the time of publication. 